\title{
COMPARISON OF THE MATCHED ASYMPTOTIC EXPANSIONS METHOD \\ AND THE TWO-VARIABLE TECHNIQUE*
}

\author{
BY \\ M. BOUTHIER \\ Centre National de la Recherche Scientifique, France
}

\begin{abstract}
Either the matched asymptotic expansions method or the two-variable technique are available for treating boundary layer problems. A comparison of the two methods is achieved on dealing with elliptic boundary value problems. The two-variable technique is proved to be slightly more powerful than the matched expansions method. Nevertheless it fails to determine a closed class of approximate solutions. Such a class, which involves the results of both the asymptotic methods is set out with help of an asymptotic equivalence theorem.
\end{abstract}

1. Introduction. The method of matched asymptotic expansions (hereafter called the M.A.E. method) and the multiple scales technique are established procedures known since the beginning of the century. As the former was discovered and developed when dealing with boundary layer problems, so the latter provided relevant material for treating "secular" problems (and other problems within unbounded domains). However one can seek to use the same multiple scales technique to treat boundary layer problems: as a matter of fact, this can be done for either ordinary differential equations (Kuen Tam, Erdelyi $[9,5])$ or partial differential equations (Comstock, Smith $[3,16])$. Certain extensions for non-linear equations have also been investigated (Searl [15]). A survey of the multiple scales technique and its applications can be found in Nayfeh [12] or in Kevorkian and Cole [8].

We are now interested in perfecting the multiple scales technique and thus extending its field of applications; this requires the analysis of the very process of the method, its foundation and its justification (Lagerstrom and Casten [10]). In this connexion, Reiss has studied the accuracy of the approximate solutions when using two or more scales; Levine and Lubot [11] have looked for optimal approximations and for optimal time scales. These two latter papers are concerned with differential equations. Otherwise, Rosenblat [14] has found the conditions for which singular perturbation problems fall to be asymptotically equivalent, viz. conditions for which the solutions of these problems are asymptotically

* Received July 8, 1982. 
equivalent (some non-linear problems are included among these). Finally, in the case of second order ordinary differential equations, Wollkind [13] has pointed out the differences which arise between the expanded solution provided by the M.A.E. method and the one due to the multiple scales technique.

In a previous paper, a study of a two-variable technique (hereafter called the T.V. technique) and its justification have been carried out for elliptic operators (Bouthier [2]): there it is proved that a weakened "secular" hypothesis permits getting a whole set of two-variable approximate solutions. Now, using the M.A.E. method, one can make out a composite expansion which typically depends on the frame of coordinates (see Kaplun [7]). So, by varying these coordinates one can also define a whole set of two-variable asymptotic approximations. The aim of the present paper is to study and to compare the approximate solutions obtained either by means of the M.A.E. method or the T.V. technique; the main results are set in Theorem 2; first, every matched asymptotic approximate solution can be found with help of the T.V. technique (the converse proposition does not always hold); next, the approximate solutions provided by one method or the other can be very simply characterized by means of common algebraic features; in fact one can define a related asymptotic equivalence class which involves and gathers all the previous approximations. Finally, it is proved that the T.V. technique itself does not always suffice to determine the whole of that class.

The elliptic boundary value problem is formulated in Sec. 2, and in turn the procedures of the M.A.E. method and of the T.V. technique are applied to it. In Sec. 3, a theorem is stated for testing an asymptotic equivalence of any two approximate solutions; hence, an asymptotic equivalence relation can be defined within a functional space which contains the approximate solutions. The main theorem is stated and proven in Sec. 4. Finally (Sec. 5 ), it is noted that this work extends certain results of Wollkind and that the asymptotic validity of approximations sets can be directly established in the case of second order operators.

\section{The two asymptotic methods.}

2.1. Formulation of the problem. Let $\Omega$ be an open bounded set of $R^{n}, \Gamma$ its boundary, $\nu$ the normal euclidean distance from $\Gamma$, and $f$ a defined function on $\bar{\Omega}$ (closure of $\Omega$ ). The following Dirichlet problem is considered:

$$
\begin{cases}(\varepsilon A+B) f=g, & \mathbf{x} \in \bar{\Omega}, \\ f, \frac{\partial f}{\partial \nu}, \ldots, \frac{\partial^{k-1} f}{\partial \nu^{k-1}} & \text { prescribed on } \Gamma .\end{cases}
$$

The letters $A, B$ stand for linear partial differential operators the coefficients of which can depend upon $\mathbf{x}$; the operator $A$ is uniformly elliptic in $\bar{\Omega}$, and its order is $2 k$. The order of $B$, say $m$, is assumed to be strictly less than $2 k$. The function $g$ depends on $\mathbf{x}$ alone and $\varepsilon$ is a small positive parameter.

Then, let $\mathbf{D}$ be the symbolic gradient vector

$$
\left(\frac{\partial}{\partial x_{1}}, \frac{\partial}{\partial x_{2}}, \ldots, \frac{\partial}{\partial x_{n}}\right)
$$


the operators $A$ and $B$ can be written down as sums of their homogeneous differential parts:

$$
A=\sum_{i=1}^{2 k} P_{i}(\mathbf{x}, \mathbf{D}), \quad B=\sum_{i=1}^{m} Q_{i}(\mathbf{x}, \mathbf{D}) .
$$

Here $P_{i}(\mathbf{x}, \cdot)$ and $Q_{i}(\mathbf{x}, \cdot)$ stand for homogeneous polynomial of degree $i$. By the very definition of ellipticity, there exists a positive constant $c_{1}$ such that:

$$
(-1)^{k} P_{2 k}(\mathbf{x}, \mathbf{p}) \geqslant c_{1}|\mathbf{p}|^{2 k}
$$

holds good for any $\mathbf{p} \in R^{n}, \mathbf{x} \in \bar{\Omega}$. For it, there also exists two constants $c_{2}>0, c_{3} \geqslant 0$ such that for any infinitely differentiable function $\varphi$ with compact support in $\Omega$ :

$$
(A \varphi, \varphi)>c_{2}\|\varphi\|_{k}^{2}-c_{3}\|\varphi\|_{0}^{2} \quad \text { (Garding's inequality) }
$$

Here $(\cdot, \cdot),\|\cdot\|_{0},\|\cdot\|_{k}$ are respectively the scalar product in $L^{2}$, the norm in $L^{2}$, and the norm in $H^{k}$. In order to illustrate our purpose, we shall below employ two simple examples in $R^{2}\left(x_{1}=x, x_{2}=z\right)$;

EXAMPLE 1 .

$$
\left\{\begin{array}{l}
\varepsilon\left(\frac{\partial^{2} f}{\partial x^{2}}+\frac{\partial^{2} f}{\partial z^{2}}\right)+\frac{\partial f}{\partial z}=0 \\
f=h \text { prescribed on } \partial \Omega
\end{array}\right.
$$

This problem which has been studied by Van Harten [17] (with $\Omega$ being a disc), is related to the parallel flow of a conducting fluid along a pipe in the presence of a uniform magnetic field. Here, we get:

$$
\begin{gathered}
n=2, \quad k=m=1, \\
P_{2}=-\left(p_{1}^{2}+p_{2}^{2}\right), \quad P_{1}=P_{0}=0, \quad Q_{1}=-p_{2}, \quad Q_{0}=0 .
\end{gathered}
$$

EXAMPLE 2.

$$
\left\{\begin{array}{l}
\varepsilon\left(\frac{\partial^{2}}{\partial x^{2}}+\frac{\partial^{2}}{\partial z^{2}}\right)^{2} f+\chi f=0, \quad \chi>0, \\
f, \quad \frac{\partial f}{\partial \nu} \quad \text { prescribed on } \partial \Omega .
\end{array}\right.
$$

This little more artificial problem will also be considered owing to its "strong" singularity when $\varepsilon \rightarrow 0(k=2, m=0, n=2)$.

Now let the Dirichlet problem (1) be required to admit a unique solution when $\varepsilon$ is small. A condition sufficient for that can be obtained by assuming either (see Example 2):

$$
\exists \text { constant } c_{4}>0:(B \varphi, \varphi) \geqslant c_{4}(\varphi, \varphi), \quad \forall \varphi,
$$

or (see Example 1):

$$
c_{3}=0 \text { and }(B \varphi, \varphi) \geqslant 0, \forall \varphi .
$$

In both cases, one can get the inequality:

$$
\exists c_{5}>0: \quad((\varepsilon A+B) \varphi, \varphi) \geqslant c_{5}\|\varphi\|_{k}, \quad \forall \varphi
$$


when $\varepsilon$ is small enough: it ensures existence and unicity of the solution $f$ if $\Gamma$ and the coefficients of (1) are sufficiently smooth (refer to Friedman). Finally in order to avoid free boundary layers we also require $\Omega$ to be strictly $B$-convex.

2.2. The matched asymptotic expansions method. A simple expansion, called "outer expansion of $f$ ", is firstly considered as:

$$
\hat{F}^{(0)}(\mathbf{x})+\delta \hat{F}^{(1)}(\mathbf{x})+\delta^{2} \ldots, \quad \delta=\varepsilon^{1 /(2 k-m)} .
$$

It is directly substituted in (1) and the resulting problem is expanded in power serie of $\varepsilon$ : that yields a hierarchy of boundary value problems which permits us to determine the unknown functions $\hat{F}^{(1)}, \hat{F}^{(2)}, \ldots$ However, the boundary condition of (1) cannot hereby be wholly satisfied and one must therefore distinguish a part ${ }^{1} \Gamma_{b}$ of $\Gamma$ along which a boundary layer occurs (the determination of $\Gamma_{b}$ and of the suitable boundary conditions for (4) are not within the scope of this paper and will be omitted here).

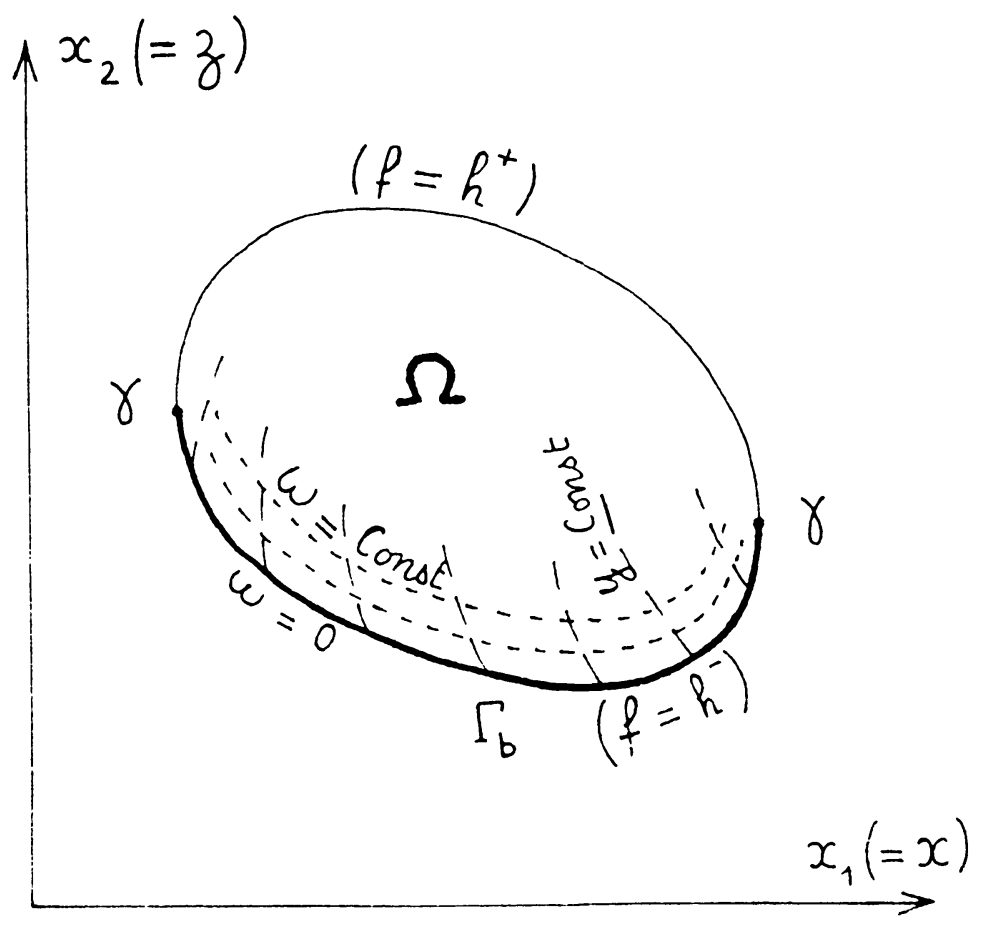

Fig. 1. Frame of curvilinear coordinates used in M.A.E. method: -- lines $\omega=$ const, ... lines $y=$ const (between brackets notations used in Example 2).

\footnotetext{
${ }^{1}$ Indeed several main boundary layers may be present, each of them being spread along a part of $\Gamma$; in the present work, without loss of generality, we focus our attention on only one of them; $\Gamma_{b}$ will be the part of $\Gamma$ which is related to that boundary layer.
} 
In order to approximate $f$ in the boundary layer, one introduces a set of curvilinear coordinates (see Fig. 1):

$$
\omega(\mathbf{x}), y_{1}(\mathbf{x}), \ldots, y_{n-1}(\mathbf{x}),
$$

where $\omega$ vanishes whenever $\mathbf{x} \in \Gamma_{b}$. No conditions are imposed on $y_{1}, y_{2}, \ldots, y_{n-1}$ but (5) forms a frame of reference within a neighbourhood of $\Gamma_{b}$. A so-called "inner variable" is then defined as:

$$
\boldsymbol{\sigma}=\delta^{-1} \omega(\mathbf{x}),
$$

and by the chain rule for derivatives, we set:

$$
\mathbf{D}=\delta^{-1} \mathbf{q}(\partial / \partial \boldsymbol{\sigma})+\mathbf{M} \cdot \mathbf{D}_{y},
$$

where $\mathbf{q}$ is the gradient $\mathbf{D} \omega ; \mathbf{D}_{y}$ stands for the symbolic gradient vector

$$
\left(\frac{\partial}{\partial y_{1}}, \frac{\partial}{\partial y_{2}}, \ldots, \frac{\partial}{\partial y_{n-1}}\right)
$$

and $\mathbf{M}$ is the rectangular matrix of the derivatives $\partial y_{j} / \partial x_{i}$. Multiplying (7) by itself, one can get higher derivative formulas, and the operators $A, B$ can then be calculated. We find:

$$
\begin{aligned}
& A=\sum_{s=0}^{2 k} \delta^{-2 k+s} V_{s}\left(\mathbf{D}_{y}\right) \frac{\partial^{2 k-s}}{\partial \sigma^{2 k-s}}, \\
& B=\sum_{s=0}^{m} \delta^{-m+s} W_{s}\left(\mathbf{D}_{y}\right) \frac{\partial^{m-s}}{\partial \sigma^{m-s}},
\end{aligned}
$$

where $V_{s}(\cdot), W_{s}(\cdot)$ stand for polynomials of degree $s$ with coefficients depending upon $w$. As a special case, we note that:

$$
V_{0}\left(\mathbf{D}_{y}\right)=P_{2 k}(\mathbf{q}), \quad W_{0}\left(\mathbf{D}_{y}\right)=Q m(\mathbf{q})
$$

But $\omega=\delta \sigma$, therefore $\mathbf{q}, \mathbf{M}$ and every coefficient $v(\omega, \mathbf{y})$ of $V_{s}$ (or $\left.W_{s}\right)$ must be expanded in powers series of $\varepsilon$, as:

$$
\begin{gathered}
\mathbf{q}(\omega, \mathbf{y})=\mathbf{q}_{b}(\mathbf{y})+\delta \sigma\left(\frac{\partial \mathbf{q}}{\partial \omega}\right)_{b}(\mathbf{y})+\delta^{2} \ldots, \\
\mathbf{M}(\omega, \mathbf{y})=\mathbf{M}_{b}(\mathbf{y})+\delta \boldsymbol{\sigma}\left(\frac{\partial \mathbf{M}}{\partial \omega}\right)_{b}(\mathbf{y})+\delta^{2} \ldots, \\
v(\omega, \mathbf{y})=v_{b}(\mathbf{y})+\delta \sigma\left(\frac{\partial v}{\partial \omega}\right)_{b}(\mathbf{y})+\delta^{2} \ldots
\end{gathered}
$$

Here $q_{b}, M_{b}, v_{b},(\partial q / \partial \omega)_{b}, \ldots$ are respectively the boundary values of $q, M, v, \partial q / \partial \omega, \ldots$ on $\Gamma_{b}$. It should be noted that every function with suffix $b$ can be fixed as constant along curves $y=$ constant and can be thereby considered as well-defined in the whole neighbourhood of $\Gamma_{b}$ where the frame of reference (5) is defined. One can insert these expansions into $(\mathcal{\complement})$ and replace $f$ by the following "inner expansion":

$$
\bar{F}^{(0)}(\sigma, \mathbf{y})+\delta \bar{F}^{(1)}(\sigma, \mathbf{y})+\delta^{2} \ldots
$$


This again leads to a hierarchy of problems; up to the first order, $F^{(0)}$ is required to satisfy:

$$
P_{2 k}(\mathbf{q})_{b} \frac{\partial^{2 k} \bar{F}^{(0)}}{\partial \sigma^{2 k}}+Q_{m}(\mathbf{q})_{b} \frac{\partial^{m} \bar{F}^{(0)}}{\partial \sigma^{m}}= \begin{cases}0, & \text { if } m \neq 0, \\ g, & \text { if } m=0 .\end{cases}
$$

where the suffix $b$ keeps the same meaning as above.

Of course, boundary conditions are missing to complete the determination of the approximate solution: it is known that they are provided partly by the so-called "matched conditions"; these conditions will not be written down here because they are not relevant to our purpose (for more details, one can refer to Eckhaus [4]). Then, adding the outer expansion and the inner one, subtracting the "common part", one can form a uniformly valid expansion which is called the "composite expansion". It must be noted that the resulting expansion actually depends upon the choice of the coordinates (5), as the inner one does. As a matter of fact, this can be exploited to make out the most relevant inner expansion: for instance, an inner expansion which is uniformly valid in the outer region: this is nothing more than the very definition of the "optimal coordinates" (Kaplun [7]).

Nevertheless, let the frame (5) vary: one obtains a whole set of first order approximations for $f$ : we shall denote this set by $A_{\mathrm{MAE}}^{(0)}$. Because of (4) and (11), every function of this set has the following form:

$$
\alpha(\mathbf{x})+\sum_{j} \beta_{j}(\mathbf{y}) \exp \left[\mu_{j}(\mathbf{y}) \sigma\right],
$$

where $\beta_{j}(i=1,2, \ldots, J)$ are defined functions which are determined by the boundary conditions (1) and by the matched conditions; $\mu_{j}(j=1,2, \ldots, J)$ are the roots with negative real part of:

$$
\mu^{2 k-m}=-Q_{m}(\mathbf{q})_{b} / P_{2 k}(\mathbf{q})_{b},
$$

and $\alpha(\mathbf{x})$ is the outer approximate part $\left(=\hat{F}^{(0)}(\mathbf{x})\right)$.

2.3. The two-variable technique. The idea of the T.V. technique is to introduce directly into the approximation an additional scaled variable, say:

$$
\tau=\delta^{-1} \theta(\mathbf{x}),
$$

which is treated as a new independent variable. Here, the function $\theta(\mathbf{x})$ is not defined for the present time but vanishes on $\Gamma_{b}$ :

$$
\theta=0, \quad \forall \mathbf{x} \in \Gamma_{b} .
$$

As pointed out in the previous paper (Bouthier), the function $\theta$ needs to be well-defined but on a neighbourhood of $\Gamma_{b}$.

In fact, the definition (14) is very close to (6), but this time, instead of (7) one performs within (1) the following substitution:

$$
\mathbf{D} \theta \delta^{-1} \mathbf{p} \frac{\partial}{\partial \tau}+\mathbf{D}, \quad \mathbf{p}=\mathbf{D} \boldsymbol{\theta} .
$$

Owing to this, a two-variable expansion can be directly sought for $f$ as:

$$
F^{(0)}(\mathbf{x}, \tau)+\delta F^{(1)}(\mathbf{x}, \tau)+\delta^{2} \ldots
$$


Up to the first order, an ordinary differential equation is derived for $F^{(0)}$ :

$$
P_{2 k}(\mathbf{p}) \frac{\partial^{2 k} F^{(0)}}{\partial \tau^{2 k}}+Q_{m}(\mathbf{p}) \frac{\partial^{m} F^{(0)}}{\partial \tau^{m}}= \begin{cases}0, & \text { if } m \neq 0, \\ g, & \text { if } m=0,\end{cases}
$$

as well as boundary conditions that we shall not write down here. One may notice that equations (18) and (11) have the same form but most often are really different: their coefficients are indeed equal only on $\Gamma_{b}$. The solutions are obtained for $F^{(0)}$ as:

$$
F^{(0)}=a(\mathbf{x})+\sum_{i} b_{i}(\mathbf{x}) \exp \left[\lambda_{i}(\mathbf{x}) \tau\right]
$$

where $a, b_{i}(i=1,2, \ldots, I)$ are functions depending on $\mathbf{x}$ alone, and $\lambda_{i}(i=1,2, \ldots, I)$ are the roots with negative real part of:

$$
\lambda^{2 k-m}=-Q_{m}(\mathbf{p}) / P_{2 k}(\mathbf{p}) .
$$

At this stage, the function $a(x), b_{i}(\mathbf{x})$ (and $\theta(\mathbf{x})$ too) remain undetermined, except on $\Gamma_{b}$ where their boundary values are prescribed according to the first order expansion of boundary conditions. Hence, one must continue by carrying out study of higher orders and get equations satisfied by $a, b_{i}$ and $\theta$; a "secular hypothesis" is then introduced and namely is: "Every approximate function $F^{(i)}$ and its derivatives must be uniformly bounded when $\tau \in[0,+\infty]$ and $x \in \Omega$ ". With the help of this hypothesis, a boundary value problem can be obtained for $a(\mathbf{x})$ (as identical to the "outer limit" of (1)), but otherwise $b_{i}(\mathbf{x}), \theta(\mathbf{x})$ remain definitely undeterminate: these latter functions can be defined arbitrarily: provided that $\theta(\mathbf{x})$ vanishes on $\Gamma_{b}$, and provided that $b_{i}(\mathbf{x})(i=1,2, \ldots, I)$ have the prescribed boundary values. By varying $\theta$ and $b_{i}(i=1,2, \ldots, I)$ one can define a whole set of two-variable approximate solutions; in the forthcoming sections, let this set be called $A_{\mathrm{TV}}^{(0)}$.

\section{An asymptotic equivalence theorem.}

3.1. Statement of the theorem. Set $\Omega, \Gamma_{b}, \gamma=\partial \Gamma_{b}$ and let consider the functions:

$$
F(\mathbf{x})=a(\mathbf{x})+\sum_{i \in I} b_{i}(\mathbf{x}) \exp \left[\delta^{-1} u_{i}(\mathbf{x})\right], \quad x \in \bar{\Omega},
$$

such that

(i) I is a finite set,

(ii) $a, b_{i}, u_{i}$ are defined $C^{1}$-continuous functions on $\Omega-\gamma$,

(iii) $u_{i}$ vanishes on $\Gamma_{b}$, and its real part elsewhere is strictly negative, $\forall i$,

(iv) $b_{i}$ and the real part of $\partial u_{i} / \partial \nu$ nowhere vanish on $\Gamma_{b}, \forall i$,

(v) if $i \neq j$, then $\partial u_{i} / \partial \nu \neq \partial u_{j} / \partial \nu$ on $\Gamma_{b}, \forall i, \forall j$.

It should be noted that the third assumption bounds the exponential terms and fixes the location of the boundary layer; the fourth condition removes from (21) terms which would be smaller than $O(1)$; and owing to the fifth condition, two "equivalent" exponential terms cannot appear together in (21) (see below). 
Of course, the defined set of functions (21) involves the previous approximations (12) and (19). Let introduce the following definition:

Definition. Any two functions $F, F^{\prime}$ such as (21) will be called "first-order equivalent" (or "equivalent") if, for any $v(\gamma)$ being a neighbourhood of $\gamma$,

$$
F^{\prime}(\mathbf{x})-F(\mathbf{x})=O(\delta) \text { holds uniformly on } \bar{\Omega}_{\gamma}=\{\mathbf{x}, \mathbf{x} \in \bar{\Omega}, \mathbf{x} \notin v(\gamma)\} .
$$

We now want to prove the following theorem:

THEOREM 1. Any two functions:

$$
\begin{gathered}
F(\mathbf{x})=a(\mathbf{x})+\sum_{i \in I} b_{i}(\mathbf{x}) \exp \delta^{-1} u_{i}(\mathbf{x}), \\
F^{\prime}(\mathbf{x})=a^{\prime}(\mathbf{x})+\sum_{j \in J} b_{j}^{\prime}(\mathbf{x}) \exp \delta^{-1} u_{j}^{\prime}(\mathbf{x}),
\end{gathered}
$$

which both satisfy the conditions (22) are first-order equivalent if, and only if:

(i) $a=a^{\prime}$,

(ii) to every $i \in I$, there is related one and only one $j \in J$, such that $\forall \mathbf{x} \in \Gamma_{b}$ :

$$
b_{i}(\mathbf{x})=b_{j}^{\prime}(\mathbf{x}), \quad D u_{i}(\mathbf{x})=D u_{j}^{\prime}(\mathbf{x}) .
$$

3.2. Proof of the direct condition. Before proving the direct condition, one must remark that: if $\phi(\mathbf{x}), \psi(\mathbf{x})$ are two defined $C^{1}$-continuous functions on $\bar{\Omega}-\gamma$ (in $\bar{\Omega}$ and outside $\gamma$ ) which both vanish on $\Gamma_{b}$, and if $(\partial \psi / \partial \nu)$ nowhere vanish on $\Gamma_{b}$, then the ratio $\phi \psi^{-1}$ can be extended as a continuous function on $\Gamma_{b}$ and its value on $\Gamma_{b}$ is equal to $(\partial \phi / \partial \nu)$. $(\partial \psi / \partial \nu)^{-1}$ (de l'Hôpital's rule). As an obvious consequence, it follows that the ratio $\phi \psi^{-1}$ is bounded on $\bar{\Omega}_{\gamma}$ whatever the neighbourhood $v(\gamma)$ being stated.

Now to prove the direct condition, one has only to demonstrate that:

$$
b_{j}^{\prime}(\mathbf{x}) \exp \delta^{-1} u_{j}^{\prime}(\mathbf{x})-b_{i}(\mathbf{x}) \exp \delta^{-1} u_{i}(\mathbf{x})=O(\delta), \quad \forall x \in \bar{\Omega}-\gamma
$$

holds good whenever the condition (24(ii)) is fulfilled. In this case, let (25) be written down as:

$$
\left(b^{\prime}-b\right) \exp \delta^{-1} u+b^{\prime}\left(\exp \delta^{-1} u^{\prime}-\exp \delta^{-1} u\right)=O(\delta)
$$

(from now on we drop the indexes $i$ and $j$ ) and let the terms of (26) be considered in turn: the first one can be set equal to:

$$
\delta \cdot \frac{b^{\prime}-b}{u} \cdot \delta^{-1} u \exp \delta^{-1} u .
$$

Owing to condition (22(iii-iv)), there exists a positive constant $\xi$ (that only depends on $v(\gamma))$ such that $z=\delta^{-1} u$ is within the sector:

$$
\pi / 2+\xi \leqslant \operatorname{Arg} z \leqslant 3 \pi / 2-\xi
$$

when $\mathbf{x} \in \bar{\Omega}_{\gamma}$. Then (27) is surely not greater than $O(\delta)$, because

(i) the function $z \exp z$ is bounded on the whole sector (28),

(ii) the function $\left(b^{\prime}-b\right) u^{-1}$ is otherwise bounded for $(22$, iv) and de l'Hôpital rule. 
Similarly, one can consider the second term of (26) as equal to:

$$
\pm \delta \cdot b^{\prime} \cdot \frac{1-\exp \delta^{-1} w}{\delta^{-1} w} \cdot \frac{w}{v^{2}} \cdot \delta^{-2} v^{2} \exp \delta^{-1} v
$$

with

$$
\begin{array}{lll}
v=u, & w=u^{\prime}-u, & \text { whenever } \operatorname{Re}\left(u^{\prime}-u\right)<0 ; \\
v=u^{\prime}, & w=u-u^{\prime}, & \text { whenever } \operatorname{Re}\left(u^{\prime}-u\right) \geqslant 0 .
\end{array}
$$

The very definition of $w$ forces $Z=\delta^{-1} w$ to stay within the half-plane:

$$
\pi / 2 \leqslant \operatorname{Arg} Z \leqslant 3 \pi / 2 \text {. }
$$

Hence (29), too, is not greater than $O(\delta)$ since:

(i) the function $(1-\exp Z) Z^{-1}$ is bounded on the whole of the previous half plane,

(ii) the function $w v^{-2}$ is bounded on $\Omega_{\gamma}$ for de l'Hôpital's rule (in the present case, the rule must be applied twice),

(iii) the function $z^{2} \exp z$ is bounded on the whole sector (28).

This completes the direct proof of Theorem 1, because if (27) and (29) are both not greater than $O(\delta)$, then (26) or (25) indeed hold.

3.3. Proof of the converse condition. Whenever $F$ and $F^{\prime}$ are equivalent, let $\delta$ tend towards zero with $\mathbf{x}$ being first fixed, the limit value of (23) is:

$$
a(\mathbf{x})=a^{\prime}(\mathbf{x}), \quad \mathbf{x} \in \bar{\Omega}, \mathbf{x} \notin \Gamma_{b} \cup \gamma .
$$

For continuity of $a$ and $a^{\prime}$, this leads to the condition (24(i)).

Otherwise, within (23), one can replace every exponential term by the following simple equivalent:

$$
b \exp \delta^{-1} u=b \exp \delta^{-1} \lambda \nu+O(\delta), \quad \lambda=\partial u / \partial \nu,
$$

and keeping $\rho=\delta^{-1} \nu$ as fixed, one can make $\delta$ tend towards zero (that namely is an "inner" limit); it yields:

$$
\sum_{i \in I} b_{i b} \exp \lambda_{i} \rho-\sum_{j \in J} b_{j b}^{\prime} \exp \lambda_{j}^{\prime} \rho=0, \quad \forall \rho \geqslant 0
$$

where $b_{i b}, b_{j b}^{\prime}$ respectively are the boundary values of $b_{i}, b_{j}^{\prime}$ on $\Gamma_{b}$.

Since (31) and its successive first derivatives with respect to $\rho$ vanish, a linear homogeneous system can be formed with respect to the unknown quantities $\left\{\exp \lambda_{i} \rho\right.$, $i \in I\} \cup\left\{\exp \lambda_{j}^{\prime} \rho, j \in J\right\}$. The determinant of this system simply is

$$
\prod_{i \in I} b_{i b} \cdot \prod_{j \in J} b_{j b}^{\prime} \cdot \prod_{\substack{p, q \in \in I \cup J \\ p \neq q}}\left(\lambda_{p}-\lambda_{q}\right) .
$$

To ensure that non-zero valued solutions really exist, one must cancel this determinant; but $b_{i b}, b_{j b}^{\prime}$ do not vanish because of (22(iv)), and any two functions $\lambda_{p}, \lambda_{q}$ are quite different whenever they both belong to $F$ (or respectively to $F^{\prime}$ ). Thus, it is required that to every $i \in I$, there is related one $j \in J$ (and only one for (22(v))) such that

$$
\lambda_{i}=\lambda_{j}^{\prime}
$$


Bringing this back into (31), and arguing in a very similar way, one can obtain:

$$
b_{i b}-b_{j b}^{\prime}=0 \text {. }
$$

This proves the desired conditions (24(ii)) and completes the proof of the Theorem 1 .

3.4. Definition. The previous theorem permits the definition of a very tractable equivalence relation between the functions (21), and by connexion one can consider the related equivalence classes. In particular let us focus attention on the approximate solutions (12) and (19): all of them ought to belong to the same equivalence class if the M.A.E. method and/or the T.V. technique are quite correct; actually, every condition of (24) was fulfilled incidentally: first, the "outer" part of (12) or (19) remains the same, whatever the asymptotic method being used; secondly the prescribed boundary value for $b_{i}(\mathbf{x})$ is the same as the one for $\beta_{i}(\mathbf{x})$; and thirdly, we have on $\Gamma_{b}$ :

$$
\begin{aligned}
& \mathbf{D}\left(\mu_{i} \sigma\right)=\sigma \mathbf{D} \mu_{i}+\mu_{i} \mathbf{q}=\mu_{i} \mathbf{q}, \\
& \mathbf{D}\left(\lambda_{i} \tau\right)=\tau \mathbf{D} \lambda_{i}+\lambda_{i} \mathbf{p}=\lambda_{i} \mathbf{p},
\end{aligned}
$$

with

$$
\begin{aligned}
& P_{2 k}(\mathbf{q})_{b}+\mu_{i}^{2 k-m} Q_{m}(\mathbf{q})_{b}=0, \\
& P_{2 k}(\mathbf{p})_{b}+\lambda_{i}^{2 k-m} Q_{m}(\mathbf{p})_{b}=0,
\end{aligned}
$$

so that $\mathbf{D}\left(\mu_{i} \sigma\right)$ and $\mathbf{D}\left(\lambda_{i} \tau\right)$ are equal on $\Gamma_{b}$, since they are colinear and have the very same magnitude, viz.

$$
|\mathbf{D} u|^{2 k-m}=P_{2 k}(\boldsymbol{\nu}) \cdot Q_{m}(\boldsymbol{\nu})^{-1}
$$

(here $\nu$ stands for the unit vector normal to $\Gamma_{b}$ ).

At this stage, one can notice that the equation:

$$
P_{2 k}(\mathbf{D} u)_{b}+Q_{m}(\mathbf{D} u)_{b}=0, \quad \forall x \in \Gamma_{b},
$$

holds good, whatever asymptotic method is used, and/or whatevery asymptotic approximation is considered.

All the approximations (12) and (19) are therefore included in the same equivalence class: this class will be called subsequently the set $A^{(0)}$.

\section{Comparison of the two methods.}

4.1. Statement of a theorem. In order to give an account of comparison between the M.A.E. method and the T.V. technique, one can state the following theorem.

TheOREM 2. Let $f$ be the solution of the Dirichlet problem (1), and let $A_{\mathrm{MAE}}^{(0)}, A_{\mathrm{TV}}^{(0)}$ and $A^{(0)}$ be the sets of first order approximate solutions which are provided respectively by the M.A.E. method, by the T.V. method and by the Theorem 1; then

$$
A_{\mathrm{MAE}}^{(0)} \subset A_{\mathrm{TV}}^{(0)} \subset A^{(0)}
$$

and moreover:

(i) if $2 k-m \leqslant 2$, then $A_{\mathrm{TV}}^{(0)}=A^{(0)}$,
if $2 k-m \geqslant 3$, then $A^{(0)} \not \subset A_{\mathrm{T}}^{(0)}$

(ii) if $2 k-m \leqslant 2 n-2$, then in most cases: $A_{\mathrm{MAE}}^{(0)}=A_{\mathrm{TV}}^{(0)}$, if $2 k-m \geqslant 2 n-1, A_{\mathrm{TV}}^{(0)} \not \subset A_{\mathrm{MAE}}^{(0)}$. 
4.2. Proof of (36). Owing to Sec. 3.4 , it is already proven that

$$
A_{\mathrm{TV}}^{(0)} \subset A^{(0)} \quad\left(\text { and } A_{\mathrm{MAE}}^{(0)} \subset A^{(0)}\right)
$$

In order to complete the proof of (36), let us now consider any coordinate frame such as (5) and the related matched asymptotic approximation (12):

$$
\begin{aligned}
& \omega(\mathbf{x}), y_{1}(\mathbf{x}), \ldots, y_{n-1}(\mathbf{x}), \\
& F^{(0)}=\alpha(\mathbf{x})+\sum_{j} \beta_{j}(\mathbf{y}) \exp \mu_{j}(\mathbf{y}) \boldsymbol{\sigma} .
\end{aligned}
$$

One wants to derive the same approximation solution $F^{(0)}$ by means of the T.V. technique; for in doing this, one can specify the function $\theta(\mathbf{x}), b_{i}(\mathbf{x})$ in the following way:

$$
\begin{aligned}
\theta(\mathbf{x}) & =\omega(\mathbf{x}), \\
b_{i}(\mathbf{x}) & =\beta_{i}(\mathbf{y}), \quad \forall i .
\end{aligned}
$$

This choice is quite possible since $\omega$ vanishes on $\Gamma_{b}$ and since $\beta_{i}$ have the required boundary value on $\Gamma_{b}$. Then the Eqs. (13) and (20) imply:

$$
\lambda_{i}=\mu_{i}, \quad \forall i
$$

so that a two-variable approximate solution is yielded which is merely identical with the matched asymptotic approximation (12). This proves that:

$$
A_{\mathrm{MAE}}^{(0)} \subset A_{\mathrm{TV}}^{(0)} \text {. }
$$

4.3. Converse proof of (ii). Conversely, set any two-variable approximation such as (19):

$$
F^{(0)}=a(\mathbf{x})+\sum b_{i}(\mathbf{x}) \exp \lambda_{i}(\mathbf{x}) \tau .
$$

The first component of the frame (5) can be chosen to be $\theta(\mathbf{x})$. Consequently the M.A.E. method provides an approximation in which each exponent $\mu_{i} \omega$ is respectively equal to each exponent $\lambda_{i} \theta$.

It remains now to find out coordinates $\mathbf{y}$ such that the functions $\beta_{i}(\mathbf{y})$ equal the functions $b_{i}(\mathbf{x})$; but $b_{i}$ and $\beta_{i}$ are already known to have the same boundary value on $\Gamma_{b}$, hence one wants to keep the functions $b_{i}$ constant wherever $\mathbf{y}$ is constant. Since there are $(n-1)$ components $\mathbf{y}$, the rank of the matrix $\partial b_{i} / \partial x_{j}$ ought to be not greater than $(n-1)$, everywhere:

$$
\operatorname{Rank}\left[\partial b_{i} / \partial x_{j}\right] \leqslant(n-1) .
$$

If this holds good, one can consider all the functions $b_{i}(1 \leqslant i \leqslant p)$ which are independent and choose the first components of $\mathbf{y}$ as being these latter functions, themselves:

$$
y_{1}=b_{1}(\mathbf{x}), \quad y_{2}=b_{2}(\mathbf{x}), \ldots, y_{p}=b_{p}(\mathbf{x}), \quad y_{p+1}(\mathbf{x}), \ldots, y_{n-1}(\mathbf{x}) .
$$

Then, owing to this background definition, every function $b_{i}$ obviously depends upon $\mathbf{y}$ alone. Although this process is correct but the frame (5) is well defined, viz. the wronskian:

$$
\frac{D\left(\theta, b_{1}, b_{2}, \ldots, b_{p}, y_{p+1}, \ldots, y_{n-1}\right)}{D\left(x_{1}, x_{2}, \ldots, x_{n}\right)}
$$

nowhere vanishes in the neighbourhood of $\Gamma_{b}$; it requires the rank of the matrix $\left(\mathbf{D} \theta, \mathbf{D} b_{1}, \ldots, \mathbf{D} b_{p}\right)$ to be equal to $p+1$ :

$$
\operatorname{Rank}\left(\mathbf{D} \theta, \mathbf{D} b_{1}, \ldots, \mathbf{D} b_{p}\right)=1+\operatorname{Rank}\left(\mathbf{D} b_{i}\right) \text { on } \Gamma_{b} .
$$


It should be noted that this needs to hold good only on $\Gamma_{b}$ : by means of continuity, validity is thereby ensured within a whole neighbourhood of $\Gamma_{b}$. Since the vector $\mathbf{D} \theta$ is normal to $\Gamma_{b}$, the same condition can also be written down as:

$$
\operatorname{Rank}\left(\mathbf{D}_{\Gamma} b_{i}\right)=\operatorname{Rank}\left(\mathbf{D} b_{i}\right) \text { on } \Gamma_{b}
$$

where $\mathbf{D}_{\Gamma} b_{i}$ is the tangential component of $\mathbf{D} b_{i}$.

If this latter condition (42) is fulfilled, one can always specify the remaining components $y_{i}(i>p)$ provided one respects the non vanishing of (41); thus, (40) (42) are necessary and sufficient to obtain the approximation (19) by using the M.A.E. method.

Now let $2 k-m \leqslant 2 n-2$; since there are at most $[(2 k-m+1) / 2]$ functions $^{2} b_{i}$, (40) is always satisfied and "usually" so is $(42)^{3}$; then, one can assert that:

$$
A_{\mathrm{MAE}}^{(0)}=A_{\mathrm{TV}}^{(0)} \text {. }
$$

Nevertheless, it can occur that for special cases, (42) no longer holds good (one can construct such particular Dirichlet problems); in these cases, the inclusion (39) must be kept strict. In the same way, it can be conversely shown that, whenever $(2 k-m) \geqslant 2 n-1$, the inclusion (39) remains strict. For instance, let us consider the example 1 of Sec. 2.1 $(2 k-m=1<2 n-2=2)$ the boundary layer lies along the inferior part or $\Gamma$. When using the MAE method one must introduce a frame of curvilinear coordinate:

$$
\sigma=\varepsilon^{-1} \omega(x, z), \quad y=y(x, z),
$$

with:

$$
\omega=0 \quad \text { on } \Gamma_{h} .
$$

The MAE procedure yields the equation "inner" equations as:

$$
\left(\omega_{x}^{2}+\omega_{z}^{2}\right) \frac{\partial^{2} F}{\partial \sigma^{2}}+\omega_{z} \frac{\partial F}{\partial \sigma}=0,
$$

hence the following inner approximations are set out:

$$
F=\beta(y) \exp \left(-\omega_{z} \sigma /|\mathbf{D} \omega|^{2}\right) .
$$

On the other hand, the T.V. technique is based on using the scale $\tau=\varepsilon \theta(x, z)(\theta=0$ on $\Gamma_{b}$ ) and it provides

$$
F=b(x, z) \exp \left(-\frac{\theta_{z}}{|D \theta|^{2}} \tau\right) \quad \text { (inner part of the approximate solution). }
$$

These approximate solutions are the very same. For evidence, one can choose

$$
\begin{array}{ccc}
\tau=\sigma, & \sigma=\tau, \\
b(x, z)=\beta(y), & \text { or } & \beta(y)=b(x, z) .
\end{array}
$$

\footnotetext{
${ }^{2}$ The integer part of $(2 k-m+1) / 2$, see Bouthier [1, p. 17].

${ }^{3} \mathrm{~A}$ sufficient condition to ensure that (42) holds good is that the $(2 k-m)$ vectors $\mathbf{D}_{\Gamma} b_{i}$ are linearly independent; in the opposite case, there exist functions $b_{i}$ which invalidate the requirement $(42)$, and $A_{\mathrm{TV}}^{(0)} \not \subset A_{\mathrm{MAE}}^{(0)}$.
} 
Thus the conclusion of the theorem holds quite good. Nevertheless one can choose the coordinate $y$ for satisfying the last equation unless $(\theta, y)$ does not form a valid frame of coordinate, viz.

$$
\frac{\partial \theta}{\partial x} \frac{\partial b}{\partial z}-\frac{\partial \theta}{\partial z} \frac{\partial b}{\partial x}=0 \text { on } \Gamma
$$

It is equivalent to

$$
b \text { is constant on } \Gamma_{b} \text {. }
$$

This can occur but when the difference between values of $h$ on the upper and the lower part of $\Gamma$ equals a constant (see Fig. 1):

$$
h_{+}(x)-h_{-}(x) \text { is constant. }
$$

If so it is, let $H(\omega)$ be any non constant function with $H(0)=h_{+}(x)-h_{-}(x)$, the T.V. approximations:

$$
F=H(\omega) \exp \left(-\omega_{z} \sigma /|D \omega|^{2}\right),
$$

can never be obtained with help of the M.A.E. method.

Furthermore, considering the Example 2 of Sec. $2.1(2 k-m=4>2 n-1=3)$ and using the M.A.E. method, one can find out the (inner) approximate solutions

$$
\begin{aligned}
F & =\left(\beta_{1}(y) \cos \delta^{-1} u+\beta_{2}(y) \sin \delta^{-1} u\right) \exp \left(-\delta^{-1} u\right), \\
\delta^{-1} u & =\varepsilon^{-1 / 4} \sigma /|\mathbf{D} \omega|^{4} .
\end{aligned}
$$

While according to the T.V. technique one can consider the (inner) approximations:

$$
\begin{aligned}
F & =\left(b_{1}(x, z) \cos \delta^{-1} v+b_{2}(x, z) \sin \delta^{-1} v\right) \exp \left(\delta^{-1} v\right), \\
\delta^{-1} v & =\varepsilon^{-1 / 4} \frac{\tau}{|\mathbf{D} \theta|^{4}} .
\end{aligned}
$$

Obviously the latter approximations cannot be equalled to the formers when:

$$
\frac{\partial b_{1}}{\partial x} \frac{\partial b_{2}}{\partial z}-\frac{\partial b_{1}}{\partial z} \frac{\partial b_{2}}{\partial x} \neq 0 .
$$

Thus the T.V. technique is more powerful in the present case than the M.A.E. method.

4.4. Converse proof of (i). In order to complete proving the theorem, one must deal with the following last proposition:

$$
A^{(0)} \subset A_{\mathrm{TV}}^{(0)} .
$$

First, let the case $2 k-m \leqslant 2$ be considered: every approximation $\in A^{(0)}$ involves only one boundary term; let it be set down as:

$$
F^{(0)}=a(\mathbf{x})+b(\mathbf{x}) \exp \delta^{-1} u(\mathbf{x}) .
$$

One wants to find out that same approximate solution by means of the T.V. technique; at once, one can keep the same factor $b(\mathbf{x})$ since the T.V. technique leaves this factor standing undeterminate. Next, in order to get the exponent $u$, a function $\theta(\mathbf{x})$ must be sought so as to satisfy: 


$$
\left\{\begin{array}{l}
u^{2 k-m} P(\mathbf{D} \theta)+\theta^{2 k-m} Q(\mathbf{D} \theta)=0 \text { in } \Omega_{\gamma}, \\
\theta=0 \quad \text { on } \Gamma_{b}
\end{array}\right.
$$

(below the suffices are omitted from $P$ and $Q$ ). But that is a Cauchy problem of the first order, and it can be seen to be homogeneous and characteristic (if $\theta_{0}$ is one solution, $C \theta_{0}$ is another solution whenever $C$ is constant). Thus to improve formalism, let us introduce the function:

$$
h=\log \left(\theta u^{-1}\right)
$$

(on $\Gamma_{b}, \log \theta u^{-1}$ is extended as continuous function: there, one defines its value as equal to $\log |\mathbf{p}||\mathbf{D} u|^{-1}$ ).

The problem (44) is thus replaced by one equation alone:

$$
P(\mathbf{D} u+u \mathbf{D} h)+Q(\mathbf{D} u+u \mathbf{D} h)=0, \quad \forall x \in \bar{\Omega},
$$

where terms can be expanded as:

$$
\begin{aligned}
& P(\mathbf{D} u+u \mathbf{D} h)=P(\mathbf{D} u)+u \mathbf{D} h \cdot \mathbf{R}(\mathbf{D} u, u \mathbf{D} h), \\
& Q(\mathbf{D} u+u \mathbf{D} h)=Q(\mathbf{D} u)+u \mathbf{D} h \cdot \mathbf{S}(\mathbf{D} u, u \mathbf{D} h) .
\end{aligned}
$$

Here $\mathbf{R}$ and $\mathbf{S}$ stand for vectors the components of which are polynomials of degree $m+1$ and $m$ respectively with respect to $(\mathbf{D} u, u \mathbf{D} h)$; when $u \mathbf{D} h$ vanishes, the values of $\mathbf{R}, \mathbf{S}$ are noticed to be:

$$
R(\mathbf{D} u, 0)=\nabla P(\mathbf{D} u), \quad S(\mathbf{D} u, 0)=\nabla Q(\mathbf{D} u) .
$$

Now, (45) is written as

$$
[R(\mathbf{D} u, u \mathbf{D} h)+S(\mathbf{D} u, u \mathbf{D} h)] \cdot \mathbf{D} h+u^{-1}[P(\mathbf{D} u)+Q(\mathbf{D} u)]=0 .
$$

This holds good whenever $u \neq 0$, but due to the very definition of $A^{(0)}$ (see (35)), the second term of (47) can be extended as a continuous function on $\Gamma_{b}$; hence (47) is a partial differential equation of first order with respect to $h$ and it is valid on the whole set $\bar{\Omega}-\gamma$.

Thereto, for ellipticity, $\mathbf{R}+\mathbf{S}$ nowhere vanishes on $\Gamma_{b}$ :

$$
\begin{aligned}
(\mathbf{R}+\mathbf{S}) \mathbf{D} u & =[\nabla P(\mathbf{D} u)+\nabla Q(\mathbf{D} u)] \mathbf{D} u \\
& =2 k P(\mathbf{D} u)+m Q(\mathbf{D} u)=(2 k-m) P(\mathbf{D} u) \neq 0, \quad x \in \Gamma_{b} .
\end{aligned}
$$

Because of this, the value of $\mathbf{D} h$ on $\Gamma_{b}$ can be calculated if any boundary value is prescribed for $h$ : thus, imposing a boundary value of $h$, one gets a Cauchy problem and this Cauchy problem is no longer characteristic: there is therefore a whole neighbourhood of $\Gamma_{b}$ where a solution of (44) can be found out to yield the required approximation (43).

Of course all this process holds good because (43) involves only one exponential term: whenever there are two or more exponential term (namely when $2 k-m>2$ ) the function $\theta(\mathbf{x})$ ought to be required to satisfy two or more equations like (44); since there is no solution to such a system, one can easily exhibit an approximation $\in A^{(0)}$ which cannot be made out by means of the T.V. technique. For instance, let us consider the Example 2 of Sec. 2.1; owing to Theorem 1, the approximate solutions

$$
\begin{gathered}
b_{1}(x, z) \cos \left(\delta^{-1} u_{1}\right) \exp \left(-\delta^{-1} u_{1}\right)+b_{2}(x, z) \sin \left(\delta^{-1} u_{2}\right) \exp \left(-\delta^{-1} u_{2}\right) ; \\
u_{i}=0, \quad \partial u_{i} / \partial \nu=1, \quad \text { on } \Gamma_{b}
\end{gathered}
$$


are known to be quite correct whatever being the functions $b_{i}, u_{i}$. When comparing these ones with the T.V. approximate solutions (see previous section), one cannot derive them from previous results when $u_{1} \neq u_{2}$.

5. Conclusion and asymptotic validity. One can use either the M.A.E. method or the T.V. technique to deal with singular perturbation of boundary value elliptic problem: these methods both provide relevant results and Theorem 2 defines requirements for their equivalence: at least, in the simple case $2 k-m \leqslant 2, n \geqslant 2$, each of them actually yields the whole set $A^{(0)}$. When including greater generality, one can assert that the T.V. technique appears to be a little bit more powerful than the M.A.E. method: as a matter of fact, there are certain approximate solutions which are yielded using the T.V. technique and which cannot be found out with the help of the M.A.E. method. This, apart from the case of the simple boundary layer $(2 k-m \leqslant 2)$, even using the T.V. technique, one fails to obtain all of the approximations $\in A^{(0)}$ : to get them, one must indeed use Theorem 1 .

In the field of ordinary differential equations, the present work is quite satisfactory for treating linear Sturm Liouville problems: it can then be considered as extending the comparison previously carried out by Wollkind.

Finally, one should remark that: first, whenever $A$ is a second order operator, the asymptotic validity of any approximation $\in A^{(0)}$ directly results from Bouthier (Sec. 3.2.2) and from the main condition (35): in fact, calculus of the Sec. 3.2.1, 3 still holds so good if $F \in A^{(0)}$, and the remainder ought to be estimated as being the very same one. Next, whenever the order of the operator $A$ is greater than 2, one can but use the Theorem 2: one approximate solution $\in A^{(0)}$ is needed being asymptotically valid and thence such are all the approximations which are provided by either the M.A.E. method or the T.V. technique.

\section{REFERENCES}

[1] M. Bouthier, Développements asymptotiques à deux types d'échelles et perturbation singulière de problèmes aux limites elliptiques, Paris, Thèse d'Etat, Université Paris 6, 1977

[2] M. Bouthier, The two-variable technique for singular partial differential problems and its justification, Quart. Appl. Math. 38, 263-276 (1980)

[3] G. Comstock, Singular perturbation of elliptic equations, SIAM J. Appl. Math. 20, 491-502 (1971)

[4] W. Eckhaus, Asymptotic analysis of singular perturbations, North-Holland, Amsterdam, 1979

[5] A. Erdelyi, Two-variable expansions for singular perturbations, J. Inst. Math. Applic. 4, 113-119 (1968)

[6] A. Friedman, Partial differential equations, Holt Rinchart and Winston, New York, 1969

[7] S. Kaplun, Fluid mechanics and singular perturbations, Academic Press, New York, 1967

[8] J. Kevorkian and J. D. Cole, Perturbations methods, Applied Mathematics, Springer-Verlag, New York, 1981

[9] K. Kuen Tam, On the asymptotic solution of the Orr-Sommerfeld equation by the method of multiple scales. J. Fluid Mech. 34, 145-158 (1968)

[10] P. A. Lagerstrom and R. G. Casten, Basic concepts underlying singular perturbation techniques, SIAM Review 14, 63-120(1972)

[11] L. E. Levine and E. S. Lubot, Optimal approximate solutions and time scales, SIAM J. Appl. Math. 29, 439-448

[12] A. H. Nayfeh, Perturbation methods, Wiley, New York, 1973

[13] E. L. Reiss, On multivariable asymptotic expansions, SIAM Review 13, 189-196 (1971) 
[14] S. Rosenblat, Asymptotically equivalent singular perturbation problems, Studies in Applied Mathematics, 55, 249-280 (1976)

[15] J. W. Searl, Expansions for singular perturbations, J. Inst. Maths Appl., 8, 131-138 (1971)

[16] D. R. Smith, The multivariable method in singular perturbation analysis, SIAM Review 17, 221-273 (1975)

[17] A. Van Harten, On an elliptic singular perturbation problem, in Ordinary and Partial Differential Equations, Lecture Notes in Math., Springer-Verlag, New York, 485-494, 1976

[18] D. J. Wollkind, Singular perturbation techniques: a comparison of the method of matched asymptotic expansions with that of multiple scales, SIAM Review 19, 502-516 (1977) 